\title{
Statin-induced autoimmune necrotizing myositis
}

\author{
Katarzyna Ząber, Beata Śliwowska, Tomasz Jasiński
}

J. Dietl Specialist Hospital, Małopolska Centre for Rheumatology, Immunology and Rehabilitation in Krakow, Poland

\begin{abstract}
Myositides comprise a large group of disorders involving limb muscle weakness. In differential diagnosis we have to consider idiopathic myositides, myositides associated with other diseases, and those induced by external factors, e.g. drug-induced. Statins are commonly used drugs, but many patients experience a broad spectrum of adverse effects including symptoms from skeletal muscle. Physicians should pay special attention to patients reporting muscle weakness lasting longer than 12 weeks, despite statin withdrawal, as well as other symptoms: dysphagia, disturbed grip function, elevated creatinine kinase (CK) levels and abnormal electromyography.

The reported case deals with the problem of differential diagnosis of drug-induced muscle injury, polymyositis with a recently reported myopathy - statin-induced autoimmune necrotizing myositis, related to anti-HMGCR antibodies.
\end{abstract}

Key words: statin-induced autoimmune necrotizing myositis, myositis, statins, anti-HMGCR antibodies.

\section{Introduction}

When describing muscle disorders we have to define the following terms: myalgia, myositis, myopathy and rhabdomyolysis.

Myalgia is a clinical condition of muscle pain, acute or chronic, local or generalized, with normal serum creatine kinase (CK) levels and without muscle weakness.

Myositis is a general term for the inflammation of muscles, causing muscle weakness and increased serum CK levels, usually ten times above the upper normal limit. Myopathy is progressive skeletal muscle damage accompanied by muscle weakness, sometimes paresis and absent deep tendon reflexes. Usually serum CK levels are elevated.

Rhabdomyolysis is a clinical condition caused by rapid destruction of muscle fibers (following toxic or mechanical injury) and release of their content into the bloodstream. Serum CK level is substantially increased, usually above $10000 \mathrm{IU} / \mathrm{I}[1,2]$.

Inflammatory muscle disorders can be divided into: idiopathic inflammatory myopathies, idiopathic inflam- matory myopathies associated with connective tissue disease, idiopathic inflammatory myopathies associated with cancer, and other forms of myopathy [3].

The present case belongs to the group of inflammatory idiopathic myopathies, which is divided into four main groups: polymyositis (PM), dermatomyositis (DM), immune-mediated necrotizing myopathy (IMNM) and inclusion body myositis (IBM). Common features of inflammatory myopathies are muscle weakness, tiredness and muscle atrophy [3, 4]. Differential diagnosis of myositis is crucial for further patient management.

Broad statin use in primary and secondary cardiovascular prevention needs careful analysis of the number of patients with potential complications. There is a broad spectrum of symptoms related to statin myopathy - from mild, self-limiting ones to fulminant rhabdomyolysis. In most cases adverse effects are mild and usually disappear after statin withdrawal.

Recently many studies have focused on IMNM, also known as necrotizing autoimmune myopathy (NAM) [5] and statin-induced autoimmune necrotizing myositis, related to anti-HMGCR antibodies (3-hydroxy-3-methyl- 
glutaryl-CoA reductase) - an enzyme involved in the synthesis of cholesterol. Recent studies indicate that anti-HMGCR antibodies are highly specific for diagnosis of IMNM [5-7].

\section{Case report}

A 64-year-old woman consulted a primary care physician because of muscle weakness lasting for several months and tiredness. The patient believed her symptoms were due to chronic exertion.

She was treated for hypercholesterolemia with statin for the last two years. The patient was diagnosed for hypothyroidism (controlled) and hiatal hernia. She also had peptic ulcer with perforation 20 years earlier. The patient apart from statin was also treated with levothyroxine $37.5 \mu \mathrm{g} /$ day and pantoprazole $20 \mathrm{mg} /$ day. Routine lab tests performed every year (blood cell count, alanine and aspartate transferase (ALT, AST), creatinine, glucose, creatine kinase, urine analysis) were normal. Lipid profile was within the normal range. Physical examination revealed mild muscle weakness of lower limbs (Lovett manual muscle testing [MMT] grade 4), statin was withdrawn, and lab tests were ordered. Lab test results from January 2015 showed elevated CK 9205 IU/I, ALT 157 IU/I, AST 176 IU/I. Complete blood count (CBC), kidney function tests, urine analysis as well as erythrocyte sedimentation rate (ESR) and C-reactive protein (CRP) level were within the normal range. Despite statin withdrawal muscle weakness progressed to Lovett MMT grade 3. The patient was referred to a neurologist and a rheumatologist. The neurology consultant did not diagnose any central nervous system disorders and referred the patient to the Emergency Department of a University Hospital in Krakow with suspicion of dermatomyositis. She was not admitted to hospital but was scheduled for admission to the Department of Neurology in April 2015. Further lab tests ordered by a primary care physician revealed (compared to earlier results) lower CK values (7758 IU/I) and similar transferases values (AST 155 IU/l, ALT 157 IU/I). Because clinical symptoms suggested dermatomyositis the patient was referred to the Department of Rheumatology.

At the time of admission the patient's general appearance and vital signs were normal. Musculoskeletal examination revealed severe muscle weakness, especially in the lower extremities (Lovett MMT grade 3), and she was not able to perform squat-stand test. Laboratory tests ordered at admission to the department revealed an elevated CK level (6705 IU/I) and also elevated levels of ALT (157 IU/I) and AST (159 IU/l). The antinuclear antibody (ANA) level was borderline positive, while other results - leucocyte and erythrocyte blood count, gamma-glutamyltransferase and thyroid, TSH, protein electrophoresis, glucose, electrolytes and urine analysis - were normal.

Due to the borderline value of ANA the myositis profile of antibodies was examined, with the following results: borderline level of anti-TIF1, low level of PM-Scl75. Other typical DM/PM autoantibodies were negative.

Elevated lipid values were observed: total cholesterol $6.38 \mathrm{mmol} / \mathrm{l}, \mathrm{LDL} 4.3 \mathrm{mmol} / \mathrm{l}$ and triglycerides within the normal range. Due to suspected dermatomyositis in differential diagnosis several diagnostic procedures were performed to exclude paraneoplastic syndrome. Abdominal ultrasonography revealed simple liver and kidney cysts and anechogenic structure of the pancreas. Computed tomography (CT) of the abdomen confirmed the ultrasonographic findings and did not reveal any neoplastic changes. Gastroscopy confirmed the diagnosis of hiatal hernia and did not reveal any other pathology. A test was performed for Helicobacter pylori confirmation, but there was no infection. Gynecological examination was normal.

Electromyography (EMG) detected primary muscle damage with pathologic resting activity - a result suggesting myositis. Muscle biopsy revealed degenerativeregenerative changes with some lymphocytic infiltration, which may suggest drug-induced muscle damage. Histopathology did not confirm clear features of myositis.

The diagnosis of polymyositis was established - suspected statin-induced autoimmune myopathy.

In order to confirm the diagnosis, a test for presence of antibodies against HMGCR was performed in Wieslab Laboratory, Malmo; anti-HMGCR level was $280 \mathrm{U} / \mathrm{ml}$ (normal values: $<13 \mathrm{U} / \mathrm{ml}, 13-20 \mathrm{U} / \mathrm{ml}$ - borderline positive, $>20 \mathrm{U} / \mathrm{ml}$ positive).

Final diagnosis: statin-induced necrotizing autoimmune myopathy (NAM) associated with autoantibodies against HMGCR.

\section{Results of treatment}

The patient has been treated with pulse steroid therapy (up to a dose of $1500 \mathrm{mg}$ methylprednisolone) i.v., with some improvement of the patient's condition and lab test results - CK level to $2745 \mathrm{IU} / \mathrm{l}$, AST $85 \mathrm{IU} / \mathrm{I}, \mathrm{ALT}$ $128 \mathrm{IU} /$. Methotrexate therapy was started (15 mg p.o. per week) and $4 \mathrm{mg}$ p.o. methylprednisolone daily.

After 4 weeks the patient came to the rheumatologist's office - she had not experienced any improvement in her condition. In physical examination there was still marked muscle weakness, Lovett MMT grade 2. Lab test results: CK 3279 IU/I, AST 78 IU/I, ALT 92 IU/l. The dose of methotrexate was increased to $20 \mathrm{mg}$ per week, and the patient was scheduled for the next appointment after 4 weeks.

At the next visit the patient showed significant improvement in her condition and improvement on 
physical examination (MMT grade 4). Lab test results: CK 1922 IU/I, AST 73 IU/I, ALT 64 IU/l. She still remains in the rheumatologist's care.

After 6 months of treatment the CK level was still elevated (760 IU/l), while ALT and AST levels were normalized. The full recovery of muscle strength was observed (MMT grade 5).

The levels of ANA and anti-HMGCR antibodies were not tested.

\section{Discussion}

Statins are broadly prescribed drugs. There are substantial benefits from their use. There is however a group of patients reporting adverse effects of various kinds. The typical toxic effect of statins is dependent on dose and length of treatment. There are some risk factors related to muscle damage: female gender, age (especially above 80 years old), low body mass index, patients with diabetes mellitus, hypertension, untreated hypothyroidism, and renal or hepatic disease, polytrauma, perioperative period, severe conditions (hypothermia, acidosis, hypoxia) viral infections (influenza, Epstein-Barr, Coxsackie) and interactions with other drugs (e.g. imidazole-based antifungals, macrolides antibiotics, fibrates or omeprazole [8]). Symptoms of statin side effects are various in nature, most often mild - muscle and tendon pain, cramps. Most severe and sometimes fulminant is rhabdomyolysis with a highly elevated CK level (above $10000 \mathrm{IU} / \mathrm{l}$ ), seen in about $0.01 \%$ of patients. Statin-related myopathy is characterized by symptom resolution after statin withdrawal [9-11].

For the last few years there have been several reports of cases of statin-induced myopathy without resolution of symptoms after treatment has been stopped. The largest group was observed in the Johns Hopkins Myositis Center (Baltimore, USA) between 2002 and 2011. Patients were referred because of muscle weakness and elevated CK levels. Among 1006 patients with suspected myopathy a group of 55 with a specific antibody was selected. $72.7 \%$ of them reported statin treatment (present or in the past). Symptoms did not resolve despite drug withdrawal. In the muscle biopsy of most patients histology revealed necrotic changes, dominant in $70 \%$ of patients, to a lesser extent in others [12-15].

In 2010 there appeared the first reports of discovery of a new particle, which was an antibody against HMGCoA reductase, the enzyme which catalyzes the reaction of HMG-CoA and mevalonic acid in the de novo pathway of cholesterol. Statins are HMG-CoA reductase inhibitors, and are used to block the complex biosynthesis of cholesterol $[14,16]$.

In order to confirm specificity of antibodies against HMG-CoA reductase, an analysis of another group was performed. This was a group of patients taking statin in primary or secondary prevention, including patients with familial hypercholesterolemia, as well as with adverse effects. The study confirmed that patients with mild myopathy, with well-tolerated treatment or mild, self-limiting myopathy do not produce antibodies against HMG-CoA reductase. On the other hand, patients with progressive muscle weakness, muscle pain, significantly elevated CK levels and with antibodies may have autoimmune myopathy, which requires immunosuppressive treatment $[15,17,18]$. Some studies indicate that $37-62 \%$ of patients with anti-HMGCR have not been treated with statins, yet presented symptoms characteristic for autoimmune myositis $[15,17]$.

Based on the regular pattern of symptoms, history of statin treatment, common features of lab tests highly elevated CK levels, myogenic muscular damage confirmed by EMG, biopsy revealing lack of or minimal inflammation, focal necrosis and presence of antibodies indicating that an autoimmune mechanism is involved - a new disorder was established, i.e. statin-induced autoimmune necrotizing myositis, as a new entity in the group of autoimmune myositides. Suggested treatment includes immunosuppressive drugs - high doses of steroids (up to $60 \mathrm{mg}$ methylprednisolone per day) as well as methotrexate, cyclophosphamide, immunoglobulins, and rituximab $[19,20]$. Some patients require plasmapheresis. Most patients respond well to therapy, but anti-HMGCR antibodies do not disappear despite normal CK levels and normal muscle strength. Some patients after immunosuppressive treatment withdrawal report return of symptoms [5, 11-13, 16, 21-23].

Based on the publications, we may suppose that the level of anti-HMGCR antibodies does not correlate with the clinical improvement. The time needed for the CK level to normalize is unknown - probably it takes months of treatment. It is important to know that the patients with polymyositis or dermatomyositis may have anti-HMGCR antibodies, but there are no data about presence of these antibodies among the healthy population. Also some patients with no statin exposure have anti-HMGCR antibodies [24].

Immune-mediated necrotizing myopathy is still a little-known disease. Studies published so far indicate that subjects producing anti-HMGCR antibodies during statin treatment may develop autoimmune muscle disease. On the other hand, necrotizing autoimmune myopathies do not always affect subjects on statin therapy $[15,17]$.

Mechanisms of myocytes damage are not well understood. It is known that it is an autoimmune process because it benefits from immunosuppressive treatment, but lack of lymphocyte infiltration suggests a mainly humoral response [17, 25]. It has been confirmed that myo- 
cyte regeneration following activation of the immune system stimulates progressive muscle damage, despite drug withdrawal [14]. It is considered whether patients with high cardiovascular risk should continue statin treatment during immunosuppressive therapy.

There is a general principle not to stop statin therapy in patients requiring such treatment $[2,12]$. Physicians should pay special attention to patients reporting muscle weakness lasting longer than 12 weeks, despite statin withdrawal, as well as other symptoms: dysphagia, disturbed grip function, elevated CK levels and abnormal electromyography $[2,4,13,16,25]$. Such patients may require further evaluation and screening for anti-HMGCR antibodies, since they benefit from immunosuppressive therapy which inhibits muscle damage.

\section{Summary}

Presently, the testing procedures for establishing anti-HMGCR antibody level are not routinely used; yet, they may find use as a screening test for revealing the risk of developing myopathy by a patient.

In conclusion, testing for anti-HMGCR antibodies should be more available to identify patients at higher risk of developing myopathy.

The authors declare no conflict of interest.

\section{References}

1. Zwolińska G, Bodzioch M, Drabczyk R. Choroby układu nerwowo-mięśniowego. In: Choroby wewnętrzne. Szczeklik A, Gajewski P (eds.). Medycyna Praktyczna, Kraków 2011; 2023.

2. Albayda J, Christopher-Stine L. Identifying statin-associated autoimmune necrotizing myopathy. Cleve Clin J Med 2014; 81: 736-741.

3. Majdan M. Zapalenie wielomięśniowe i skórno-mięśniowe. In: Postępy reumatologii klinicznej. Zimmermann-Górska I (ed.). Wyd. Lekarskie PZWL, Warszawa 2014; 213-227.

4. Majdan M. Dylematy diagnostyczno-terapeutyczne. PM/DM czy groźna, postatynowa miopatia? Interdyscyplinarne Oblicza Reumatologii 2015; 2: 4-6.

5. Preuße C, Goebel HH, Held J, et al. Immune-mediated necrotizing myopathy is characterized by a specific Th1-M1 polarized immune profile. Am J Pathol 2012; 181: 2161-2171.

6. Werner JL, Christopher-Stine L, Ghazarian SR, et al. Antibody levels correlate with creatine kinase levels and strength in anti-3-hydroxy-3-methylglutaryl-coenzyme A reductase-associated autoimmune myopathy. Arthritis Reum 2012; 64: 4087-4093.

7. Watanabe $\mathrm{Y}$, Suzuki S, Nishimura $\mathrm{H}$, et al. Statins and myotoxic effects associated with anti-3-hydroxy-3-methylgutaryl-coenzym A reductase autoantibodies: an observational study in Japan. Medicine 2015; 94: e416.
8. Kanth R, Shah MS, Flores RM. Statin-associated polymyositis following omeprazole treatment. Clin Med Res 2013; 11: 9195.

9. Mohassel P, Mammen AL. The spectrum of statin myopathy. Curr Opin Rheumatol 2013; 25: 747-752.

10. Fulcher J, Keech A. The case for more intensive use of statins. Ther Adv Chron Dis 2012; 3: 201-210.

11. Talbert RL. Safety issues with statin therapy. J Am Pharm Assoc 2006; 46: 479-490.

12. Mammen AL. Autoimmune myopathies: autoantibodies, phenotypes and pathogenesis: Statin-associated IMNM. Nat Rev Neurol 2011; 7: 343-354.

13. Del Grande M, Christopher-Stine L. Expanding our understanding of statin myopathy. When it's autoimmune? Int I Clin Rheumatol 2012; 7: 243-246.

14. Sirvent P, Mercier J, Lacampagne A. New insights into mechanisms of statin-associated myotoxicity. Curr Opin Pharm 2008; 8: 333-338.

15. Mammen AL, Pak K, Williams EK, et al. Anti-HMG-CoA reductase antibodies are rare in statin users, including those with self-limited musculosceletal side-effects. Arthritis Care Res (Hoboken) 2012; 64: 269-272.

16. Christopher-Stine L, Casciola-Rosen LA, Hong G, et al. A novel autoantibody recognizing $200-\mathrm{kd}$ and $100 \mathrm{kd}$ proteins is associated with an immune-mediated necrotizing myopathy. Arthritis Rheum 2010; 62: 2757-2766.

17. Alshehri A, Choksi R, Bucelli R, Pestronk A. Myopathy with antiHMGCR antibodies: Perimysium and myofiber pathology. Neurol Neuroimmunol Neuroinflamm 2015; 2: e124.

18. Musset L, Miyara M, Benveniste $O$, et al. Analysis of autoantibodies to 3-hydroxy-3-methylgutaryl-coenzym A reductase using different technologies. J Immunol Res 2014; 2014: 405956.

19. Ramanathan S, Langguth D, Hardy TA, et al. Clinical course and treatment of anti-HMGCR antibody-associated necrotizing autoimmune myopathy. Neurol Neuroimmunol Neuroinflamm 2015; 2: e96.

20. Lundberg IE, Vencovsky J, Alexanderson H. Therapy of myositis: biological and physical. Curr Opin Rheumatol 2014; 26: 704-711.

21. Davidson MH. Combination therapy for dyslipidemia: safety and regulatory considerations. Am J Cardiol 2002; 90 (suppl 109): 50K-60K.

22. Mammen AL, Gaudet D, Brisson D, et al. Increased frequency of DRB 1*11:01 in anti-hydroxymethylglutaryl-coenzyme A reductase-associated autoimmune myopathy. Arthritis Care Res (Hoboken) 2012; 64: 1233-1237.

23. Bukhari M. Drug-induced rheumatic diseases. Curr Opin Rheumatol 2012; 24: 182-186.

24. Yongpeng Ge, Xin Lu, Qinglin Peng, et al. Clinical characteristics of anti-3-hydroxy-3-methylglutaryl coenzyme A reductase antibodies in Chinese patients with idiopathic inflammatory myopathies. PLoS One 2015; 10: e0141616.

25. Mohaupt MG, Karas RH, Babiychuk EB, et al. Association between statin-associated myopathy and skeletal muscle damage. CMAJ 2009; 181: E11-E18. 